\title{
Thomas Marksteiner
}

\section{Der Bonda-Survey: Archäologische Feldforschungen auf dem Territorium der ostlykischen Polis Limyra}

Der Bonda-Survey hat sich die feldarchäologische Untersuchung eines zwischen den Küstenebenen von Finike und Demre gelegenen, steil aus der See ansteigenden Höhenzuges zur Aufgabe gemacht (Abb. 1), der, wie Schriftzeugnisse belegen, zumindest in der Kaiserzeit dem Polisterritorium von Limyra zugehörte1. Dieses heute nur auf Maultierpfaden erreichbare und von Wanderhirten saisonal genutzte Hochland, durch welches einst eine in dem Stadiasmos von Patara sowie in der Tabula Peutingeriana verzeichnete Straßenverbindung von Kaunos über Telmessos nach Myra und Attaleia verlief ${ }^{2}$, war, wie zahlreiche teils ausgedehnte Niederlassungen belegen, in antiker und nachantiker Zeit dicht besiedelt. Bei der Auswahl des Bonda-Gebietes für eine archäologische Prospektion wurden folgende Kriterien vorrangig gewichtet: eine Zugehörigkeit zum Polisterritorium von Limyra, ein guter Erhaltungszustand der Baubefunde sowie eine möglichst deutliche, in der Topographie vorgegebene naturräumliche Abgrenzung ${ }^{3}$.

Schon seit Beginn der 70er Jahre des 20. Jahrhunders führten Mitarbeiter der Limyra-Grabung punktuelle Untersuchungen im Umland des Poliszentrums durch. Insbesondere J. Borchhardt und G. Stanzl unternahmen zahlreiche Begehungen, im Zuge derer sie antike Befunde beschrieben und kartierten: Die Ergeb-

\footnotetext{
1 Das Feldforschungsunternehmen wurde im Rahmen des Forschungsprogrammes der Limyra-Grabung des Institutes für Klassische Archäologie der Universität Wien durchgeführt und durch den Fonds zur Förderung der Wissenschaftlichen Forschung der Republik Österreich finanziert. Zu Vorberichten zum Bonda-Survey s. s. A. Konecny, Th. Marksteiner, in: XVI. Kazi Sonuçlari Toplantisi II (1995) 237 f.; dies., in: XVII. Kazi Sonuçlari Toplantisi II (1996) 146 f.; dies., in: XVIII. Kazi Sonuçlari Toplantisi (1997) 173 ff.; dies., in: XIX. Kazi Sonuçlari Toplantisi II (1998) 26f.; dies. ÖJh 96 (1997) Beibl. 390 ff.; A. Konecny, B. Marksteiner, Th. Marksteiner, in: XX. Kazi Sonuçlari Toplantisi II (1999) 142ff.; dies., in: XXI. Kazi Sonuçlari Toplantisi II (2000) $86 \mathrm{f}$.

2 Zum Stadiasmos von Patara s. etwa S. Şabin, Lykia 1 (1994) $130 \mathrm{ff}$. Zu dieser Wegeverbindung s. etwa TAVO BV 15.2 Lykien und Pamphylien; Verf., in: F. Kolb (Hrsg.), Lykische Studien 1. Die Siedlungskammer von Kyaneai in Lykien (Asia Minor Studienreihe 9, 1993) 97. 3 Dazu s. Verf. in: K. Belke, F. Hild, J. Koder, P. Soustal (Hrsg.), Byzanz als Raum, Denkschrift Wien 283, TIB 7 (2000) 116-118.
} 


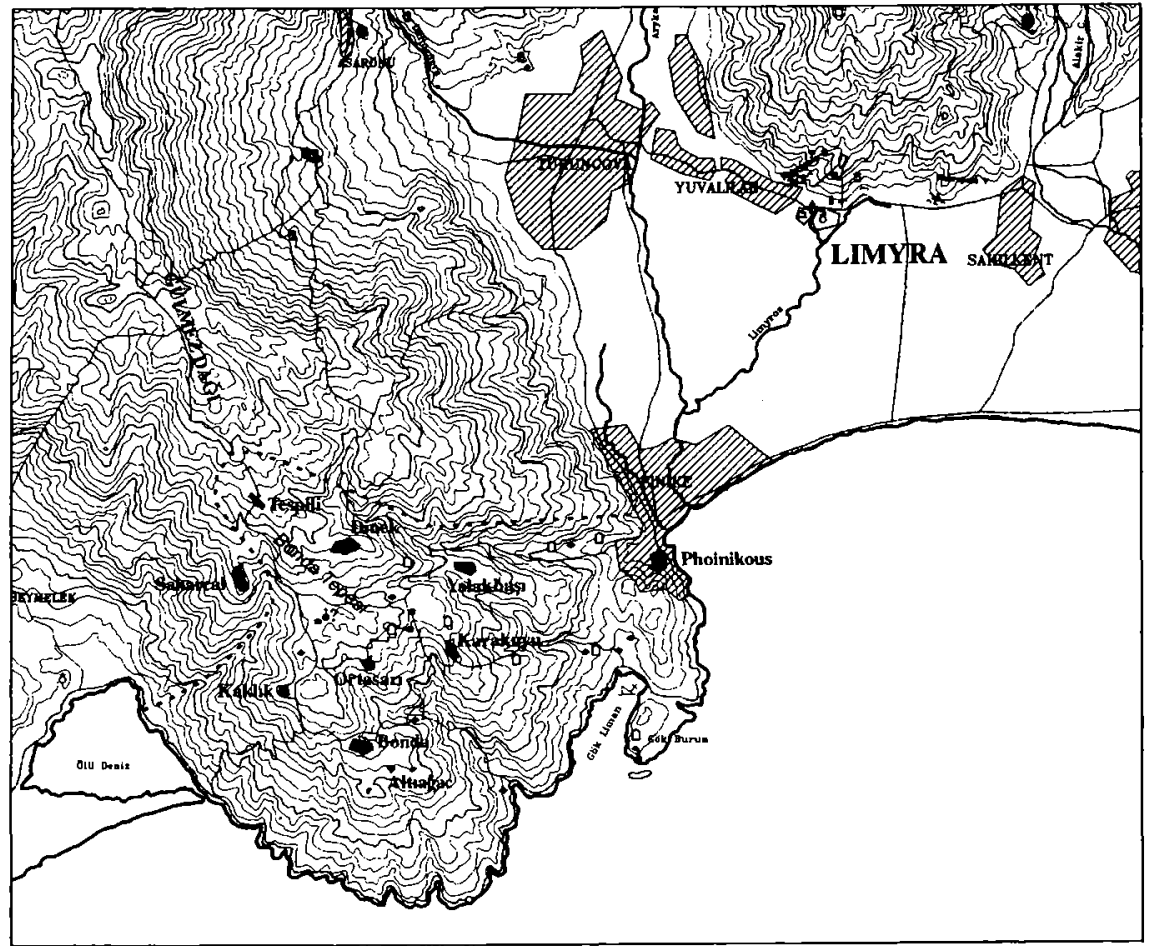

Abbildung 1

nisse dieser Surveys flossen in Karten des Territoriums und Vorberichte ein ${ }^{4}$. Der Verfasser beschäftigte sich mit einer Gruppe von Befunden, die Stanzl dem Defensivsystem der Polis zugeschrieben hatte, bei denen es sich jedoch um landwirtschaftliche Einrichtungen und Gehöfte handeln dürfte ${ }^{5}$. A. Konecny untersuchte und veröffentlichte mehrere Turmgehöfte auf dem Territorium von Limyra ${ }^{6}$. Die erste systematische Oberflächenuntersuchung wurde durch J. Koder und R. Jakobek im Westbereich des Polisterritoriums durchgeführt, wobei spätantike und byzantinische Siedlungsbefunde im Zentrum des Interesses standen, aber auch antike Bauten kartiert und teils auch durch Archäologen der Limyra-Grabung aufgenommen und veröffentlicht wurden?. Die nachantiken Siedlungsbefunde blieben jedoch weitgehend unpubliziert.

${ }^{4}$ s. etwa G. Stanzl, 7. Kazi Sonuçlari Toplantisi (1985) 440-442.

5 Verf., ÖJ 63 (1994) $95 \mathrm{ff}$.

${ }^{6}$ A. Konecny, Denkschrift Wien 235 (1993) 47 ff.; ders., Hellenistische Turmgehöfte in Zentral- und Ostlykien (1997) $15 \mathrm{ff}$.

7 s. R. Jacobek, Jahrbuch der Österreichischen Byzantinistik 42 (1992) $287 \mathrm{ff}$.; A. Konecny, Lykia 1 (1994) 124-126; Verf., in: F. Blakolmer et alii (Hrsg.), Fremde Zeiten, Festschrift für J. Borchhardt I (1996) 85. 
Das Territorium von Limyra war also zu Beginn des Bonda-Surveys im Jahr 1994 schon vergleichsweise gut bekannt. Forschungsreisende, die das abgelegene und schwierig zu erreichende Bonda-Gebiet vor Errichtung der Küstenstrasse durchquert hatten, berichteten von Turmbauten am Wege und Sarkophagen mit Inschriften ${ }^{8}$. J. Borchhardt besuchte das Gebirgsland in den 70er Jahren'; seine Berichte gaben den Anstoß zur Aufnahme der Surveyarbeiten durch den Verfasser in Zusammenarbeit mit den Archäologen A. Konecny und B. Marksteiner sowie dem Epigraphiker M. Wörrle.

\section{Die Topographie des Surveygebietes}

Das etwa $20 \mathrm{~km}^{2}$ umfassende Surveygebiet liegt in den südlichen Bereichen eines steil aus der See aufsteigenden und sich wie ein Keil zwischen die Küstenebenen von Finike/Limyra und Demre/Myra schiebenden Ausläufers des Akdag-Massives. Die nördliche Grenze des Untersuchungsgebietes wird durch ein westlich des Hafenortes Finike/Phoinikous/Phoinix gelegenes, tief eingeschnittenes Trockental angezeigt, während im Nordwesten die steil ansteigenden und hochgebirgsartigen Charakter annehmenden Höhenbereiche eine natürliche Grenze für menschliche Ansiedlung gebildet zu haben scheinen.

\section{Die naturräumlichen Grundlagen}

Der Naturraum des Bonda-Gebietes kann am besten als stark bewegte Berglandschaft charakterisiert werden, die durch Kuppen und Grate sowie dazwischen befindliche Talfurchen gegliedert wird. Die wenigen landwirtschaftlich nutzbaren Binnenebenen sind relativ kleinflächig, so daß als Nutzland vor allem Hänge zur Verfügung standen, auf denen Terrassenwirtschaft betrieben werden konnte. Die nördlichen Höhen waren wohl in der Antike wie auch heute noch dicht bewaldet und mögen forstwirtschaftlich genutzt worden sein. Im gesamten Bonda-Gebiet fehlen natürliche Fließwasservorkommen, so daß die Wasserversorgung auf $\mathrm{Zi}$ sternenwirtschaft basierte. Dadurch ergab sich zwangsläufig eine Tendenz zur Siedlungskontinuität im Bereich der in der Errichtung aufwendigen Wasserspeicher. Auch die heutigen Viehzüchter und Wanderhirten nutzen noch die antiken Zisternen und sorgen auch fallweise für deren Instandhaltung.

${ }^{8}$ T. A. B. Spratt, E. Forbes, Travels in Lycia, Milyas and Cibyratis I (1847) 143 f.; Freya Stark, Auf den Spuren Alexanders (1962) $176 \mathrm{ff} . ;$ R. Heberdey, E. Kalinka, Bericht über zwei Reisen im Südwestlichen Kleinasien, Denkschrift Wien 45 (1896) 15.

9 . Borchbardt, in: J. Borchbardt et alii, Myra. Eine lykische Metropole in antiker und byzantinischer Zeit, in: IstForsch 30 (1975) 91 und Anm. 149. 


\section{Die Siedlungslagen}

Alle größeren dörflichen Ansiedlungen des Bonda-Gebietes befinden sich in Kuppen- oder Gratlage. Dies mag zumeist mit dem wehrhaften Charakter der frühen Niederlassungen, andernfalls aber auch mit Wunsch, Nutzland von Verbauung freizuhalten, in Zusammenhang stehen. Es scheint jedenfalls ein besonderes räumliches Naheverhältnis der Dörfer zu Agrarflächen bei der Auswahl der Siedlungslagen nicht immer vorrangig gewichtet worden zu sein. Etwas anders stellt sich das Bild bei Betrachtung der in der Regel in peripheren Siedlungslagen, in unmittelbarer Nähe von agrarischem Nutzland errichteten Einzelgehöfte oder Weiler dar.

\section{Zur Zielsetzung des Bonda-Surveys}

Das Ziel der in den Jahren 1994 bis 1999 durchgeführten Untersuchungen war es, siedlungs- und wirtschaftsgeschichtliche Abläufe in einer peripheren Siedlungskammer des Polisterritoriums von Limyra zu rekonstruieren. Dabei wurden bautypologischen und siedlungstypologischen Studien sowie der Erforschung von diachronen Veränderungen der Siedlungsmuster besondere Aufmerksamkeit gewidmet. Insgesamt standen weniger Einzelmonumente, als größere Zusammenhänge im Vordergrund des Interesses. Diese konnten jedoch ohne Kenntnis von typologisch und chronologisch einordenbaren Einzelobjekten kaum erfaßt werden.

Die Grundlage unserer Arbeit bildete daher die Aufnahme der baulichen Befunde des Survey-Gebietes. Ein weiteres Desiderat, der Versuch einer Evaluierung der Anbauflächen, welche in Zusammenhang mit der Aufnahme des Baubestandes Rückschlüsse auf die wirtschaftliche Situation ländlicher Gemeinden erlauben könnte, mußte vorerst zurückgestellt werden. Einige Bedeutung kam der Klärung offener Fragen zur politischen Topographie des Bonda-Gebietes zu, wobei insbesondere die Grenzziehung zwischen den Polisterritorien von Myra und Limyra hervorzuheben ist.

\section{Zur Methode des Bonda-Surveys ${ }^{10}$}

Das Surveyunternehmen wurde mit vergleichsweise geringen Mitteln und kleinem Personalaufwand durchgeführt, wodurch methodische Einschränkungen vorgegeben waren. Insbesondere aufgrund der schwierigen Logistik erschien es ratsam, die Zahl der Mitarbeiter zu beschränken. Im Rahmen der nur achttägigen

10 s. Verf., in: K. Belke, F. Hild, J. Koder, P. Soustal (Hrsg.), Byzanz als Raum, Denkschrift Wien 283, TIB 7 (2000) $119 \mathrm{ff}$. 
Kampagnen konnten daher intensive Oberflächenbegehungen mit dem Anspruch auf ein vollständiges Erfassen der Befunde nicht unternommen werden. Dieser Mangel wurde zumindest teilweise durch Informationen seitens der lokalen Bevölkerung, zumeist Hirten, wettgemacht. Auch erlauben es Erfahrungswerte, typische Siedlungslagen zu erkennen. Als vorläufiges Ergebnis der Untersuchungen darf festgestellt werden, daß aufgrund der Siedlungskonzentration in dörflichen Agglomerationen die Zahl der Streugehöfte und Weiler vergleichsweise gering gewesen sein dürfte. Neuzeitliche Niederlassungen wurden ausschließlich in Verbindung mit älteren Siedlungsresten aufgenommen, mußten andernfalls aber aus der Untersuchung ausgeklammert bleiben.

Die entdeckten antiken, nachantiken und mittelalterlichen Befunde wurden lagemäßig bestimmt, kartiert sowie, sofern ihr Erhaltungszustand dies sinnvoll erscheinen ließ, vermessen und dokumentiert.

Da aufgrund spezifischer Bedingungen im Surveygebiet oft nur wenige datierbare und dann zumeist späte Keramikfunde gemacht wurden, bereitet die zeitliche Einordnung der Befunde besondere Probleme ${ }^{11}$. Es mußten folglich nebst dem keramologischen Befund auch alternative Ansätze herangezogen werden. Genannt seien vor allem analytische Untersuchungen zur Bautechnik und Bautypologie, wobei diesbezüglich, dank der regen Surveytätigkeit des letzten Jahrzehntes, Vergleichsmaterial aus Lykien zur Verfügung steht ${ }^{12}$. Dabei mußte jedoch eine gewisse Unschärfe in Kauf genommen werden, da auf diesem Wege bestenfalls eine grobe Zuweisung an Perioden, keinesfalls aber eine feinchronologische Differenzierung erfolgen kann. Auf die zeitliche Einordnung schlecht erhaltener Befunde mußte oft verzichtet werden. Einen weiteren Ansatz, ein relativchronologisches Gerüst zu erstellen, um sich einer Datierung anzunähern, bot das am Befund ablesbare zeitliche Verhältnis von Bauten und Bauabschnitten. Sich an mehreren Befunden und Befundgruppen wiederholende Abläufe erlaubten es, Phasen intensiver Bautätigkeit zu erfassen. Auch der epigraphische Befund lieferte chronologische Indizien. Diese sind zwar zumeist nicht direkt auf Einzelbauten projizierbar, bieten aber doch Informationen zur zeitlichen Spanne der Nutzung von Niederlassungen.

\section{Die Siedlungen des Bonda-Gebietes}

Im folgenden werden die wichtigsten Dörfer und einige Einzelgehöfte des Survey-Gebietes vorgestellt und der Versuch unternommen, anhand der jeweils erhaltenen Monumente die Geschichte der Niederlassungen nachzuzeichnen ${ }^{13}$.

$11 \mathrm{Zu}$ dieser Frage s. auch ebenda $119 \mathrm{f}$. und $F$. Kolb in diesem Band.

12 Vergleiche nur die Beiträge von $F$. Kolb, A. Sanli, U. Hailer und A. Thomsen in diesem Band.

13 Die angeführten Orte sind auf der Karte (Abb. 1) eingezeichnet, die Reihenfolge der Beschreibung führt von Ost nach West und von Nord nach Süd. 
Diese Informationen sollen die Grundlage für eine Rekonstruktion der Siedlungsgeschichte des Bonda-Gebietes bilden. An dieser Stelle muß betont werden, daß die Befundaufnahme erst 1999 abgeschlossen wurde und daher die analytische Auswertung des Materials am Anfang steht, so daß nur ein vorläufiger Ergebnisstand dargestellt werden kann.

Yalak Başı (Seehöhe: ca. 600 m; verbaute Gesamtfläche etwa: 7000 m²)

Die Ruinen im Bereich des Yalak Başı sind besonders schlecht erhalten: Der untere Bereich der Niederlassung ist durch neuzeitliche Siedlungsaktivität stark in Mitleidenschaft gezogen, während im Gipfelbereich teils gewaltige, unstrukturierte Schutthalden anstehen.

Aufgrund des extrem schlechten Erhaltungszustandes sind kaum jemals geschlossene Grundrisse erfaßbar, so daß Schätzungen bezüglich der Zahl der Hauseinheiten schwierig sind: Diese dürfte etwa bei 25 bis 30 gelegen haben.

Klassische Besiedlung wird durch Keramikfunde im Bereich der Niederlassung und in einem nahegelegen Gehöft belegt. Ihre größte Ausdehnung scheint die Siedlung während des Hellenismus und der Kaiserzeit erreicht zu haben. Etwa die gleiche Fläche wurde zu einem späteren Zeitpunkt mittels eines aus Spolien errichteten Mauerzuges befestigt. Teils noch anstehende Mauerzüge sowie zahlreiche, als Spolienblöcke verbaute bzw. in Versturzlage befindliche Werkstücke zeugen vom einstigen Vorhandensein qualitätvoller Hausteinarchitektur. Ein besonders großflächiger, einräumiger Bau im Südwesten der Siedlung darf vielleicht dem öffentlichen Bereich zugewiesen werden. Darauf weist auch der Umstand, daß in der näheren Umgebung zahlreiche faszierte Blöcke sowie von einem Stufenunterbau stammende Werkstücke in Wiederverwendung versetzt wurden. Diese stammen von einem oder mehreren, relativ aufwendig gestalteten Monumenten.

Im Weichbild der Niederlassung standen mehrere Sarkophage, die Hauptnekropole lag jedoch in einem Geländesattel im Norden, im Bereich einer Wegkreuzung. Dort befinden sich die stark zerstörten Reste von etwa zehn Grabbauten. Einige über den Südhang verstreute Gräber geben wohl den Verlauf eines weiteren Zugangsweges an. In diesem Bereich stand auch ein qualitätvolles Monument, wohl ein Heroon, von dem jedoch nur mehr verstürzte Werkstücke zeugen.

Am Yalak Başı haben sich keinerlei Hinweise auf die Existenz eines Kirchenbaus erhalten. Ein Weiterbestehen der Siedlung in nachantiker Zeit wird jedoch durch den Spolienmauerzug an der Südflanke und Keramikfunde belegt.

Karakuyu (Seehöhe: ca. 650 m; verbaute Gesamtfläche etwa $15000 \mathrm{~m}^{2}$ )

Bei dem Dorf mit rund 40 bis 50 Hauseinheiten handelt es sich um die zweitgrößte Niederlassung des Bonda-Gebietes. In Karakuyu konnte keine vorhellenistische Besiedlung nachgewiesen werden; eine hellenistische Phase ist durch Grabinschriften und Keramikfunde belegt. 
Der Ruinenbestand erlaubt es nicht, die Lage eines Ortszentrums während der antiken Siedlungsphasen zu erschließen. Deren Verbauung zog sich über die gesamte Nordsüd-Erstreckung der Niederlassung, wobei es sich an der Peripherie um einen lockeren Streuverband gehandelt haben dürfte. In diese Periode gehören meist einräumige Grundrisse, seltener sind zwei- oder dreiräumige Bauten. Häufig sind den Häusern Höfe und Terrassen vorgelagert. An der Peripherie des verbauten Geländes liegen Wirtschaftsgebäude, darunter mehrere Preßhäuser. Im Nordbereich befinden sich auf einer felsigen Erhebung Steleneinlassungen und Nischen: Hier könnte das kultische Zentrum gelegen haben. Das Gelände auf der Kuppe ist in agglutinierender Weise dicht verbaut. Unter den Befunden finden sich teils antike Hausteinbauten, teils späte Mörtelbruchsteinmauern. Es konnten keine Hinweise auf das Bestehen einer Befestigungslinie festgestellt werden.

Die Zahl der in und um Karakuyu erhaltenen Sarkophage beträgt knapp 20 Stück, wobei die zeitliche Streuung, wie Inschriften und Schmuck zu erschließen erlauben, vom fortgeschrittenen Hellenismus bis in die späte Kaiserzeit reicht. Die Gräber liegen teils entlang des Zugangsweges im Norden, teils an der Peripherie der Niederlassung; in einigen Fällen sind sie mit einer Hausanlage vergesellschaftet. Eine Konzentration befindet sich am Osthang außerhalb der Wohnverbauung.

Die größte Ausdehnung und Verbauungsdichte erreichte der Ort wohl in spätantik-frühbyzantinischer Zeit: Eine Geländeterrasse im Westen scheint damals erstmals als Baugrund genutzt worden zu sein. In einer im Zentrum des Ortes gelegenen, dreischiffigen Pfeilerbasilika fanden sich geschmückte Architekturfragmente, die Verwandtschaft mit der Bauornamentik der Anlagen von Alakilise, Muskar oder Karabel im nördlichen Hinterland von Myra aufweisen ${ }^{14}$. Hervorzuheben sind mehrere, wahrscheinlich von einem Ziborium stammende Werkstücke, die durch A. Pülz aufgrund von Vergleichen in die erste Hälfte des 6. Jahrhunderts $\mathrm{n}$. Chr. datiert werden können. Weiters gehört eine große, überdachte Zisterne nahe der Kuppe in die nachantiken Siedlungsphasen. Die in Mörtelbruchsteintechnik ausgeführten Hausbauten dieser Periode sind meist einräumig mit vorgelagertem Hof.

Die neuzeitliche Nutzung der Ruinen und vor allem der Zisternen führte zu teils nachhaltigen Veränderungen des Siedlungsbildes: Es wurden ausgedehnte Terrassen und mauerbegrenzte Freiflächen angelegt.

Ortasart (Seehöhe: ca. 750; verbaute Gesamtfläche etwa $6000 \mathrm{~m}^{2}$ )

Im Bereich von Oratasarı ist keine vorhellenistische Besiedlung nachweisbar; eine hellenistische Phase wird durch Grabinschriften belegt sowie durch bautypologische Erwägungen indiziert.

Den Kuppenbereich nimmt eine sehr schlecht erhaltene Befestigungsanlage von etwa $800 \mathrm{~m}^{2}$ Flächenausdehnung ein, an deren Mauern sich eine antike Phase und

14 s. A. Pülz, Mitteilungen zur Christlichen Archäologie 1 (1975) $62 \mathrm{f}$. 
eine späte, unter Spolienverwendung ausgeführte Erneuerung nachweisen lassen. Die Errichtung der agglutinierenden Anlage kann aufgrund der Mauertechnik und in Analogie zu Bauten in Bonda Harabesi, Dinek Tepesi und Tespili Yayla in den Hellenismus datiert werden. Um diese Kuppenbefestigung erstreckte sich die aus meist einräumigen Hausanlagen mit vorgelagerten Höfen bestehende antike Siedlung. Ein öffentliches Siedlungszentrum konnte in den Ruinen nicht ausgemacht werden. An der Peripherie der Niederlassung finden sich wiederum gehäuft landwirtschaftliche Einrichtungen.

Die nachantike Niederlassung scheint auf die Kuppe und den Westhang beschränkt gewesen zu sein, wo sich mehrere, in Mörtelbruchsteintechnik errichtete Hausanlagen um einen kleinflächigen Apsidalbau gruppieren. Solch kapellenartige Kultbauten werden in Lykien meist in die mittelbyzantinische Zeit datiert ${ }^{15}$. Eine Entstehung in Zusammenhang mit oder im Anschluß an die Instandsetzung der Gipfelbefestigung erscheint am wahrscheinlichsten. Diese wurde unter Verwendung zahlreicher Spolien der antiken Siedlung durchgeführt. Im Denkmalbestand von Ortasari fehlen neu errichtete Bauten der spätantik-frühbyzantinischen Periode; auch hat sich keine basilikale Kirche erhalten. Die Niederlassung dürfte in dieser Phase stark an Dynamik eingebüßt haben, die antike Bausubstanz war jedoch, wie Keramikfunde belegen, auch im 6. und 7. Jahrhundert n. Chr. bewohnt.

Bonda Harabesi (Seehöhe ca.: 630 m; verbaute Gesamtfläche: etwa $20000 \mathrm{~m}^{2}$ )

Im Bereich der Ruinen von Bonda Harabesi ist keine vorhellenistische Besiedlung nachweisbar; eine hellenistische Phase ist durch Grabinschriften belegt sowie durch bautypologische Erwägungen indiziert. Mit geschätzten 60 Hauseinheiten handelt es sich um die größte Niederlassung des Bonda-Gebietes.

Eine Kuppenbefestigung nimmt eine Fläche von knapp $1000 \mathrm{~m}^{2}$ ein. Sie kann aufgrund der Mauertechnik und in Analogie zu den anderen Anlagen des BondaGebietes in den frühen Hellenismus datiert werden. Es handelt sich um eine agglutinierende Anlage, bestehend aus mindesten einem turmartigen Kernbau, in den Mauerzug einbezogenen, teils auch zweiräumigen Einbauten und verbindenden Mauerzügen; das durch einen Mauerversprung gedeckte Tor öffnete sich nach Westen. Die Anlage weist eine nachantike Ausbauphase auf, im Zuge derer sie nach Osten hin erweitert wurde.

In der Niederlassung haben sich zahlreiche Hausgrundrisse der antiken Siedlungsphasen erhalten: es handelt sich um ein-, zwei- und dreiräumige Bauten, denen oft Höfe vorgelagert sind. Im verbauten Bereich befinden sich zahlreiche Zisternen. Eine Baugruppe im Zentrum der Niederlassung könnte öffentlichen

15 Geringe Größe einer Kirche und das Fehlen von Bauplastik können vielleicht als Kriterien für eine Datierung in mittelbyzantinische Zeit gewertet werden. s. U. Peschlow, in: J. Borchhardt, G. Dobesch (Hrsg.), Akten des 2. Internationalen Lykiensymposions in Wien 1990, Band 2, TAM Ergh. 18, Denkschrift Wien 235 (1993) 61 f.; C. Foss, DOP 48 (1994) 35 spricht sich für eine Zuweisung sekundärer Einbauten in basilikale Anlagen und anderer, kleinflächiger Kirchen im Myragebiet in komnenische Zeit aus. 
Charakter gehabt haben: Mehreren, in der Breite angeordneten Räumen war eine sich auf eine Hoffläche öffnende Halle vorgelagert. Das sehr qualitätvolle Mauerwerk weist auf eine Entstehung im Hellenismus oder der frühen Kaiserzeit. In diesem Bereich der Siedlung konnte auch eine auffällige Häufung von Inschriftenfunden festgestellt werden.

Knapp 40 teils auch reliefgeschmückte Sarkophaggräber haben sich in den Nekropolen der Siedlung erhalten: Die größte Konzentration findet sich im Geländesattel im Norden der Befestigung, weiters liegen mehrere Gräber am Hang südlich der Niederlassung. Im Zentrum der Wohnsiedlung sind einige Gräber mit Hausanlagen vergesellschaftet.

In spätantik-frühbyzantinischer Zeit erreichte die Niederlassung ihre größte Ausdehnung: In Mörtelbruchsteintechnik errichtete Häuser ziehen sich weit den Westhang hinab und auch der zentrale Bereich war dicht verbaut. Zwei große Kirchen entstanden in dieser Periode. In die untere Basilika wurde sekundär eine kleine Saalkirche eingebaut, so daß ein Weiterbestehen der Niederlassung in mittelalterlicher Zeit denkbar erscheint.

Altı A ğaç (Seehöhe: ca. $550 \mathrm{~m}$; verbaute Gesamtfläche: etwa $3000 \mathrm{~m}^{2}$ ).

Im Bereich südlich der Niederlassung Bonda-Harabesi findet sich eine Konzentration vorhellenistischer Befunde. In Altı Ağaç liegen die Ruinen einer vorhellenistischen, wahrscheinlich klassischen Niederlassung, die sich um einen befestigten Bau in Kuppenlage gruppiert. Die aus fünf bis sechs Hauseinheiten bestehende Ansiedlung zeichnet sich durch lockere Verbauungsstruktur aus. Im Inneren der Kuppenbefestigung fanden sich Einbauten, die teilweise sekundär sein dürften. Ein großes Steinbecken weist auf wirtschaftlichen Nutzung zu einem nicht definierbaren Zeitpunkt. Da der für vorhellenistische Herrensitze typische, turmartige Kernbau fehlt, könnte es sich bei dieser Anlage um eine Art Fluchtburg der dörflichen Gemeinschaft gehandelt haben. Die Hauseinheiten sind meist mehrräumig mit vorgelagerten Höfen und Terrassen. Eine aufwendige, halbkreisförmig an Felsen angeschobene Steinsetzung im Nordwesten des Dorfes ließe sich als Zisterne interpretieren, hydraulischer Mörtel hat sich jedoch nicht erhalten.

Es fehlen Hinweise auf nachklassische Bautätigkeit, durch eine Mühle des Trapetum-Typs ist allerdings landwirtschaftliche Verarbeitungstätigkeit für spätere Perioden gesichert. Eine Preßanlage im Osten des Dorfes entspricht dem Typus, der ab hellenistischer Zeit verbreitet war: Ihr Auffangbecken wurde zu einem späteren Zeitpunkt zur Zisterne umgearbeitet.

Im Süden des Dorfes liegt ein spätantik-frühbyzantinisches Gehöft. Dieses setzt sich aus einem Hauptbau mit angesetztem Hof und Nebenbauten zusammen und entspricht im strukturellen Aufbau vergleichbaren Anlagen etwa im IstladaGebiet auf dem Territorium von Myra ${ }^{16}$. Weiters gehörten landwirtschaftliche Einrichtungen, vielleicht Viehgehege, zur Anlage.

16 Vgl. etwa Th. Marksteiner, A. Konecny, XIV. Araştirma Sonuçlari Toplantisi II (1997) 466. 
Ein klassisches Einzelgehöft, dessen struktureller Aufbau aufgrund der schlechten Erhaltung nicht rekonstruiert werden konnte, lag etwa $250 \mathrm{~m}$ östlich von Altı Ağaç. Auf einer Kuppe südwestlich von Altı Ağaç befindet sich eine vorhellenistische Ringmauer von etwa $50 \mathrm{~m}$ Durchmesser. Es fehlen Hinweise auf Einbauten, dies mag jedoch auf die schlechte Erhaltung der Anlage zurückführbar sein, es könnte sich aber auch um eine Fluchtburg gehandelt haben.

Kaklik (Seehöhe: $750 \mathrm{~m}$; verbaute Fläche: $650 \mathrm{~m}^{2}$ )

Die kleine Befestigungsanlage von Kaklik ist sehr schlecht erhalten, der Bestand erlaubt es allerdings trotzdem, die Struktur der Anlage zu rekonstruieren und zwei Bauphasen zu erschließen: In klassischer Zeit wurde eine sich aus einem turmartigen Kernbau und einer angeschlossenen, ummauerten Fläche zusammensetzende, befestigte Anlage errichtet. Vergleichbare Burgen sind in Lykien häufig belegt und werden in der Regel als Herrensitz interpretiert ${ }^{17}$.

In einer hellenistischen Ausbauphase wurde die klassische Ringmauer nach Süden hin erweitert und damit die ummauerte Fläche um etwa ein Drittel vergrößert. Den bestehenden Mauerring verstärkte man zudem mittels des Einbaus eines Turmes. In diese Zeit darf wohl auch ein rund $50 \mathrm{~m}$ östlich der Anlage in den Fels geschlagenes Fassadenkammergrab datiert werden: Hierfür sprechen nebst der Grabinschrift in griechischer Sprache auch bautypologische Erwägungen.

Am erhaltenen Baubestand lassen sich keine Hinweise auf spätere Umbauten feststellen.

Dinek Tepesi (Seehöhe ca: 970 m; verbaute Gesamtfläche: ca. 20000 m²)

Im Bereich der Ruinen auf dem Dinek T. ist keine vorhellenistische Besiedlung nachweisbar; eine hellenistische Phase ist durch Grabinschriften und Keramikfunde belegt sowie aufgrund bautypologischer Erwägungen indiziert.

Die Kuppe des Siedlungshügels wird durch eine befestigte Anlage eingenommen. Über den Süd- und den Westhang erstreckt sich eine ausgedehnte Hangsiedlung von lockerer Verbauungstruktur, in der sich geschätzte 35 bis 40 Hauseinheiten erhalten haben. Im Westbereich, an der Peripherie des verbauten Geländes liegen dem öffentlichen Bereich zuweisbare Bauten. Die Gräber liegen über die Hänge im Süden und Westen der Niederlassung verstreut.

Die schlecht erhaltene Befestigungsanlage nimmt eine Fläche von etwa $1400 \mathrm{~m}^{2}$ ein. Es handelt sich im eine agglutinierende Anlage mit je einem kernbauartigen Turm an den Schmalseiten. Ihre Errichtung kann aufgrund der Mauertechnik und in Analogie zu dem Wehrdorf auf der Tespili Yayla in den frühen Hellenismus datiert werden. Keramikfunde aus dem Bereich der Einbauten reichen bis in das fortgeschrittene 3. Jahrhundert v. Chr. zurück und unterstützen den durch die Architektur gewonnenen Datierungsansatz. Die dichte Binnenverbauung ist teils im

17 s. etwa Verf., Die befestigte Siedlung von Limyra (1997) $144 \mathrm{ff}$. 
Verband mit der Kurtine errichtet. An den Mauern lassen sich mehrere Umbauphasen ablesen. Schlackenfunde belegen eine gewerbliche Nutzung des Areals in einer späteren Siedlungsphase.

Im Bereich südlich unterhalb der Befestigungslinie ist die Hangsiedlung extrem schlecht erhalten: Zahlreiche sorgfältig ausgeführte Werkstücke in den ausgedehnten Schutthalden belegen jedoch das einstige Vorhandensein qualitätvoller Hausteinarchitektur insbesondere auf den ersten zwei Geländeterrassen unterhalb der Zitadelle. In diesem Bereich, wohl am Aufweg zur Burg, standen auch mehrere Monumente, von denen verstreute Werkstücke zeugen. Die tiefer am Hang befindlichen Bauten sind tendenziell nachlässiger ausgeführt und dürften wohl auch späteren Siedlungsphasen zuzuweisen sein.

Auf einer schwach ausgeprägten Erhebung am Fuß des Siedlungshanges befindet sich eine Baugruppe. Diese scheint in der Kaiserzeit, im Zuge eines einheitlichen Bauprojekt errichtet worden zu sein, das dem öffentlichen Bereich zuzuordnen ist. Das Kernstück des Komplexes bildete eine kleine, zweiräumige Therme vor deren Front ein Ehrenmonument stand. An die Badeanlage schließen weitere Bauten an; einer derselben beherbergt einen Hallenraum. Natürliche Senken nördlich und östlich der Therme scheinen zu großen Wasserspeichern ausgebaut worden zu sein. Eine weitere, großflächige Zisterne mit aufgemauerter Mittelstütze liegt im Süden der Niederlassung, etwas außerhalb des verbauten Gebietes.

Im Weichbild und in der weiteren Umgebung der Niederlassung befinden sich zahlreiche Sarkophage, darunter auch einige besonders aufwendig gestaltete und skulpturengeschmückte Monumente. Ein aus zwei Sarkophagen und einem Altar bestehendes Ensemble erhebt sich auf einer kleinen Terrassenanlage oberhalb der antiken Wegtrasse.

Für die Siedlungsgeschichte des Bonda-Gebietes ist der Umstand, daß sich in den Ruinen am Dinek-Tepesi das Vorkommen von Mörtelbruchsteinmauerwerk auf einige Bauten in mittlerer Hanglage beschränkt und zumeist mit Ausbesserungen an bestehenden Gebäuden in Zusammenhang steht, von besonderer Bedeutung. Nachantike Neubauten wurden vorrangig im südöstlichen Hangbereich errichtet: Es handelt sich um zwei Hausanlagen. Das Fehlen einer Kirche unterstreicht die geringe Bedeutung der spätantik-frühbyzantinischen Siedlung.

Tespili Yayla (Seehöhe: ca. 1100 m; verbaute Gesamtfläche: etwa 1400 m²$^{2}$ )

Im Bereich der Ruinen auf der Tespili Yayla ist keine vorhellenistische Besiedlung nachweisbar; eine hellenistische Phase ist durch Keramikfunde belegt.

Die Ruinen gehören zu einer agglutinierenden, befestigten Anlage mit zwei kernbauartigen Türmen an der Angriffsseite, teils in die Ummauerung einbezogenen Einbauten und dichter Binnenverbauung. Ihre Datierung in den Hellenismus basiert auf mauertechnischen Erwägungen und Keramikfunden. Eine Deutung als Wehrdorf wird durch den strukturellen Aufbau der Anlage indiziert. Außerhalb der Ringmauer finden sich nur geringe Verbauungsspuren: Wahrscheinlich lagen an der Südostflanke am Fuß der Befestigungen eine Kultanlage und am Hang un- 
terhalb Grabbauten. Auffällig ist das Fehlen von Sarkophaggräbern. In der näheren Umgebung wurden mehrere Ölpressen und Zisternen aufgefunden.

Der Baubestand im Inneren der Befestigungen erlaubt es zwar, geringfügige, einer sekundären Bauphase zuschreibbare Umbauten abzulesen, insgesamt überwiegt jedoch der Eindruck weitgehender Homogenität der Bausubstanz. Dieser wird durch den Keramikbefund gestützr: Es überwiegt hellenistisches Material; nur wenige Scherben deuten auf das Weiterbestehen einer Restsiedlung in nachhellenistischer Zeit.

\section{Gehöftanlagen und Streubesiedlung}

Die Zahl der im Survey-Gebiet aufgefundenen Einzelgehöfte und kleinen Weiler ist relativ gering: Dies könnte mit dem Fehlen systematischer Begehungen erklärt werden, dürfte aber auch mit der Konzentration der Besiedlung in fünf großen Dörfern in Zusammenhang stehen. Mehrfach fanden sich allerdings landwirtschaftliche Einrichtungen, etwa Preßanlagen und einfache Bauten, in der Nähe von Feldfluren. Die Einzelgehöfte und Weiler liegen fallweise in der Nähe der dörflichen Siedlungen, sind aber zumeist auf periphere Fruchtkammern orientiert. In einigen Fällen bereitet die Datierung der Gehöfte Schwierigkeiten, da die bisher am lykischen Material erarbeiteten bautypologischen und mauertechnischen Datierungskriterien nicht greifen und der Keramikbefund keine zeitliche Zuweisung erlaubt. Im Folgenden sollen einige charakteristische Befunde vorgestellt werden.

\section{Gehöfte im Gießtal westlich von Finike}

Das Gießtal westlich von Finike bietet beiderseits des Trockenbettes landwirtschaftlich nutzbares, wenngleich durch felsige Abschnitte kompartimentiertes Gelände. Zahlreiche Terrassierungsmauern zeugen von einstiger landwirtschaftlicher Nutzung. In diesem Bereich fanden sich am nördlichen Hangbereich zwei nebeneinander gelegene Terrassenbauten vorhellenistischer Zeitstellung, ein hellenistisches Turmgehöft mit zugeordneter Preßanlage, welches die älteren Bauten ersetzt haben dürfte, sowie ein wohl in spätantik-frühbyzantinischer Zeit errichteter Bau, der eine Ölmühle und eine Preßanlage beherbergte.

\section{Rundturm bei Ortasarı}

Etwa $350 \mathrm{~m}$ östlich von Ortasarı haben sich die Ruinen eines aufgrund der Mauertechnik in hochklassische Zeit datierbaren Rundbaus erhalten. Er konnte über einen an der Nordwestseite befindlichen Eingang betreten werden. Es könnte sich hier um ein Turmgehöft auf kreisförmigen Grundriß handeln, wie sie für die grie- 
chischen Inseln verschiedentlich, für Lykien jedoch bisher nicht nachweisbar sind $^{18}$. Die in unmittelbarer Nähe befindlichen Fragmente eines Sarkophaggrabes dürfen als Beleg für eine Weiternutzung des Baus bis in die Kaiserzeit gelten.

\section{Befestigtes Gehöft bei Yukarı Camliyurt}

Im Bereich einer kleinen Binnenebene südwestlich des Dinek Tepesi haben sich die Ruinen eines aufgrund mauertechnischer Kriterien der hellenistischen Periode zuweisbaren, befestigten Gehöftes erhalten. Die kleinflächige, kompakte Anlage war über einen durch einen Mauerversprung links flankierten Zugang betretbar. Im Inneren scheinen eine hofartige Freifläche und an die Außenmauern angelehnte Räumlichkeiten gelegen zu haben. In unmittelbarer Nähe des Kernbaus haben sich die Ruinen eines Nebengebäudes und mehrere Preßanlagen erhalten.

\section{Überlegungen zu den landwirtschaftlichen Grundlagen der Besiedlung des Bonda-Gebietes}

Hervorzuheben sind die zahlreichen, im Bonda-Gebiet erhaltenen Preßanlagen. Diese sind häufig mit Mühlen assoziiert und dienten wohl der Ölproduktion ${ }^{19}$. Sowohl die Felspressen als auch die Mühlen waren relativ aufwendig herzustellen und bedeuteten eine beachtliche Investition. Derartige Anlagen mußten sich folglich amortisieren und wurden kaum nur für den Eigenbedarf eingerichtet ${ }^{20}$.

In Karakuyu und in der näheren Umgebung der Niederlassung fanden sich beispielsweise Hinweise auf das Vorhandensein von 14 Preßanlagen, für die A. Konecny ein Produktionspotential von 140001 per Saison errechnete ${ }^{21}$. Es handelte sich also um eine quasi-industrielle, auf den Verkauf orientierte Produktion von Olivenöl, was insbesondere durch das Vorkommen von Doppelpressen in aufwendigen Gebäuden schon für die hellenistische Periode erschlossen werden kann. Unklar bleibt, inwieweit schon in klassischer Zeit in großem Umfang Öl produziert wurde, da sich in dieser Periode zuweisbaren Baubefunden nur selten Preßanlagen befinden. Diese haben in einigen Fällen kreisförmige Preßbecken, es überwiegt jedoch der spätere, rechteckige Typ, wobei sekundäre Errichtung mög-

18 s. etwa J. H. Young, Hesperia 25 (1956) 122ff.; A. Bon, BCH 54 (1930) 147 ff.; L. Haselberger, Befestigte Turmgehöfte auf den Kykladeninseln Naxos, Paros und Keos (unpublizierte Dissertation, TU München 1978) passim.

${ }_{19}$ A. Konecny, ÖJh 67 (1998) Beibl. 121-194; ders., ÖJh 68 (1999) Beibl. 160 ff.

20 Noch heute findet man in Lykien auf die Deckung des Eigenbedarf ausgerichtete bäuerliche Betriebe, welche die alten Mahltassen sowie Trapeta oder andere Mahlsteine weiterverwenden, in denen jedoch die Bearbeitung des Preßgutes mittels der Füße in halbierten und ausgehöhlten Baumstämmen erfolgt.

21 A. Konecny, ÖJh 67 (1998) Beibl. 143. 
lich erscheint, da Gehöfte in der Regel über einen längeren Zeitraum bewirtschaftet wurden. In Mörtelbruchsteintechnik errichtete Preßhäuser belegen, daß die Ölproduktion auch noch in spätantik-frühbyzantinischer Zeit eine bedeutende Rolle spielte.

Nebst der Ölwirtschaft ist wohl auch schon für die Antike mit Weinproduktion zu rechnen. Für die frühbyzantinische Zeit bietet die Nikolaus-Vita (VNS c. 25, 55 und 63) eine Quelle für Weinanbau in vergleichbarer Lage und in unmittelbarer räumlicher Nachbarschaft zum Bonda-Gebiet ${ }^{22}$. In diesem wird seit kürzerem im Bereich der Tespili Yayla wieder Wein angebaut und die Stöcke werden mit Zisternenwasser bewässert.

Günstige Getreideanbauflächen sind im Bonda-Gebiet, in dem auch heute noch Wintergetreide angebaut wird, vor allem auf zentrale Höhenlagen und einige kleinflächige Fruchtkammern konzentriert, sonst überwiegt eher starkes Gefälle. Die sehr skeletthaltigen Böden sind insgesamt wohl wenig ertragreich. Eine Episode der Nikolaus-Vita erlaubt Einblick in die bäuerliche Lebenswelt und die Ertragslage im gebirgigen Hinterland von Myra (VNS c. 59f.): Die Ernte eines Feldes bei Arneai entsprach der Menge der Aussaat; erst durch das Eingreifen des Archimandriten erhöhte sich das Verhältnis auf 5:1, ein für die antike Landwirtschaft hervorragendes Ergebnis ${ }^{23}$. Bei den Eckzahlen der Episode dürfte es sich jedoch um Extremwerte handeln, so daß das tatsächliche Ertragsverhältnis vielleicht im Schnitt bei 3:1 gelegen haben mag.

Angesichts der allgemeinen Tendenz früher Landwirtschaft zur Diversifizierung und der reichlichen Verfügbarkeit von Weideflächen kann davon ausgegangen werden, daß im Bonda-Gebiet in antiker Zeit die Viehwirtschaft eine bedeutende Rolle gespielt hat. Für die frühbyzantinische Periode sind in der Nikolaus Vita verschiedentlich Ochsenschlachtungen überliefert (VNS c.56); an anderer Stelle wird ein Hirt mit Weidegründen in den Bergen erwähnt (VNS c.62); während einer Trockenheit fürchten die Bauern um ihre Tiere (VNS c. 20). Im SurveyGebiet läßt sich allerdings im archäologischen Befund Viehhaltung mit zwei möglichen Ausnahmen nicht direkt nachweisen: Im Weichbild der klassischen Niederlassung bei Altı Ağaç und nahe einem spätantik-frühbyzantinischen Gehöft haben sich vielleicht Gehege erhalten. Aus einer Analyse des stratifizierten tierischen Knochenmaterials der Limyra-Grabung ergab sich für die spätgeometrisch-früharchaissche Periode und die ausgehende Kaiserzeit ein signifikantes Überwiegen der Ziege gegenüber dem Schaf24. Dieses erlaubt wohl auch Rückschlüsse auf die Tierhaltung in den naheliegenden Berglandschaften.

Ausweislich des archäologischen Befundes bildete die Ölproduktion einen nicht $\mathrm{zu}$ vernachlässigenden Faktor in der Ökonomie des Bonda-Gebietes und war wahrscheinlich die Grundlage des Wohlstandes der Gemeinwesen. Sie war,

${ }^{22}$ Es wird insbesondere ein zum Sionskloster gehöriges Weingut erwähnt (VNS c. 63).

${ }^{23}$ s. M. Zimmermann, Untersuchungen zur historischen Landeskunde Zentrallykiens (Antiquitas Reihe 1, Bd. 42, 1992) 104 und Anm. 14; H. Blum, Die Vita Nicolai Sionitae (1997) $119 \mathrm{f}$.

24 G. Forstenpointner, G. Gaggl, ÖJh 66 (1967) Beibl. 424. 
wie die zahlreichen aufwendigen Preßanlagen belegen, darauf ausgelegt, nennenswerte Überschüsse zu erwirtschaften. Diese wurden wohl nicht nur in die städtischen Zentren der Region, sondern auch außerhalb Lykiens verhandelt, wie die Grabinschrift eines Reeders und Händlers in Olympos zu erschließen erlaubt, der seinen Reichtum mit dem Verkauf von Öl im Pontosgebiet gemacht haben dürfte ${ }^{25}$. Die Bedeutung der Produkte des Hinterlandes - genannt werden Nahrungsmittel und Feuerholz - für die Bevölkerung der ostlykischen Küstenstädte wird durch eine weitere Erzählung der Nikolaus Vita (VNS c.52) verdeutlicht: Aufgrund der Verweigerung der ländlichen Produzenten, den städtischen Markt zu beliefern, kam es in Myra zu einer Versorgungskrise, durch welche sich die Magistrate zum Eingreifen gezwungen sahen.

\section{Bevölkerungszahlen}

Schätzungen bezüglich der Einwohnerzahl antiker Niederlassungen und Landstriche können zumeist nur als Annäherungswerte gelten, sie bieten aber zumindest eine ungefähre Vorstellung der Größenordnungen, mit denen zu rechnen ist. Aufgrund der Zahl der erhaltenen Hauseinheiten läßt sich für die Dörfer des Bonda-Gebietes zur Zeit deren größter Ausdehnung eine Einwohnerzahl zwischen 100 und 250 Personen erschließen. Das gebirgige Hinterland von Myra betreffende Angaben in der Nikolaus Vita bewegen sich in vergleichbarer Größenordnung 26: im Dorf Plenion wurden anläßlich eines Festaktes 200 Sitzplätze eingerichtet (VNS c. 55); die männliche Bevölkerung von Tragalassos, die herbeigeeilt war, um einen Felsen zu verschieben, zählte 75 Köpfe (VNS c. 39); im Dorf Plakoma beobachteten 300 Personen die Austreibung eines bösen Geistes (VNS c. 16). Eine vorsichtige, unter Einbeziehung diachroner Verschiebungen der Siedlungsschwerpunkte erstellte Schätzung ergibt für das Bonda-Gebiet der Kaiserzeit eine Bevölkerung von etwa 800 bis 1000 Seelen.

\section{Strassen und Wege}

Die heute noch genutzten Saumpfade des Bondagebietes dürften im Großen und Ganzen dem antiken und mittelalterlichen Wegenetz entsprechen: Dies wird durch direkt mit Wegbauarbeiten in Verbindung stehende Einrichtungen, etwa Felstreppungen oder talseitige Stützmauern, sowie durch indirekte Hinweise belegt. Zu den letzteren zählen nebst topographischen Vorgaben die oft entlang der Wegverbindungen errichteten Sarkophaggräber, ein Felsrelief und Zisternen. Zu den bedeutendsten Entdeckungen des Bonda-Surveys gehört ein direkt an der

25 A. Konecny, ÖJh 67 (1998) Beibl. 147.

26 s. die Überlegungen von Blum, a. O. 13. 
erhaltenen Wegtrasse gelegenes, inschriftlich auf Straßenbauarbeiten Bezug nehmendes Monument ${ }^{27}$. Das etwa drei Meter lange Bauwerk in Gestalt eines Altars, dessen Inschrift den ersten Statthalter Lykiens, Quintus Veranius, und die Titulatur des Claudius nennt, wurde anläßlich der Provinzeinrichtung in der Mitte des ersten Jahrhunderts n. Chr. errichtet.

Die ost-west orientierte Hauptachse des Wegesystems diente nicht nur der Anbindung der dörflichen Gemeinden und dem Nahverkehr zwischen den Küstenebenen von Myra und Limyra, sondern hatte wohl auch über das lokale Aufkommen hinausgehende Bedeutung, da sie für den Landverkehr die günstigste Verbindung zwischen Ost- und Zentralykien darstellte. Aus einem inschriftlich überlieferten Erlaß zur Regulierung des Fährbetriebes zwischen Myra und Limyra wird jedoch deutlich, daß bei entsprechenden Wetterverhältnissen im Küstenbereich wohl die Schiffahrt das bevorzugte Verkehrsmittel darstellte (OGIS 572).

Während im östlichen Bereich des Survey-Gebietes der Verlauf der Hauptachse in der Topographie vorgegeben war - das Trockental hinter Finike bietet sich als Zugangsweg an - scheint sich die Wegführung an Westhang des Bonda-Gebietes in zwei Trassen geteilt zu haben. Eine Wegverbindung führte ins westliche Hochland und wahrscheinlich weiter nach Myra. Eine weitere Trasse überwindet den steilen Westhang des Bonda-Massives in Richtung der Lagune und dürfte die Küstenebene in Richtung Myra durchlaufen haben.

Weiters waren alle dörflichen Siedlungen des Bonda-Gebietes untereinander durch ein dichtes Wegenetz verbunden. Wegverbindungen führten auch an den östlichen Küstenbereich, wo sich kleine Niederlassungen befanden bzw. auch tiefe Buchten als Anlegestellen Verwendung gefunden haben dürften.

\section{Zusammenfassende Überlegungen zur Siedlungsentwicklung im Bonda-Gebiet}

Für die vorhellenistische Zeit sind folgende Siedlungstypen im Ruinenbestand des Bonda-Gebietes nachweisbar: Ein kleiner Herrensitz, eine dörfliche Niederlassung, eine Fluchtburg (?) und mehrere Einzelgehöfte. Auffällig ist das Fehlen eines bedeutenderen Herrensitzes mit entsprechenden Grabbauten bzw. einer Agglomeration von Zentralortcharakter: Man gewinnt den Eindruck, daß es sich beim Bonda-Gebiet um eine periphere Siedlungslage gehandelt haben dürfte. $\mathrm{Da}$ für spricht auch die Verteilung der Befunde, da sich die vorhellenistische Besiedlung tendenziell auf den Nahbereich der Küstenebenen bzw. auf besondere Gunstlagen konzentrierte. Auch scheinen Höhenlagen unter 500-600 m Seehöhe bevorzugt worden zu sein.

${ }^{27} \mathrm{Zu}$ diesem Monument und dem Wegesystem des Bonda-Gebietes s. Verf., M. Wörrle, Chiron 32 (2002) $545 \mathrm{ff}$. 
In frühhellenistischer Zeit erfolgte wahrscheinlich der Ausbau des klassischen Herrensitzes bei Kaklık. Die Errichtung eines erweiterten Mauerringes und eines relativ aufwendigen Felsgrabes mit Inschrift könnten als Hinweis darauf gewertet werden, daß die grundbesitzende Elite noch in dieser Periode über bedeutende Mittel und den politischen Freiraum, befestigte Anlagen zu errichten, verfügte.

In hellenistischer Zeit, wohl schon im 3. Jahrhundert v. Chr., fand im BondaGebiet eine massive Landnahme statt: Die Errichtung von vier strukturell ähnlichen und auch in der Flächenausdehnung vergleichbaren, wehrdorfartigen Anlagen entlang des Steilabfalles zur Ebene von Myra dürfte wohl einem Planungskonzept entsprochen haben und mit dem Schutz des Grenzgebietes von Limyra zur Nachbarpolis in Zusammenhang stehen. Im Fall der Niederlassung Bonda Harabesi war wohl die hellenistische Neugründung mit einem synoikismos verbunden: Das vorhellenistische Dorf bei Altı Ağaç und die frühen Gehöfte der Umgebung wurden ausweislich des archäologischen Befundes in dieser Periode aufgegeben. Um drei dieser hellenistischen Wehrdörfer scheinen sich noch in vorrömischer Zeit ausgedehnte offene Siedlungen entwickelt zu haben. Nur die höchstgelegene Niederlassung auf der Tespili Yayla vollzog diesen Entwicklungsschritt nicht mit. Die Ursache hierfür mag wohl im Mangel ertragreichen agrarischen Nutzlandes in der höhenbedingten Ungunstlage begründet gewesen sein.

Weiters dürfte in hellenistischer Zeit die dörfliche Niederlassung Karakuyu gegründet worden sein. Im Ruinenbestand haben sich keine Befestigungen erhalten, so daß man davon ausgehen darf, daß es sich um eine offene Siedlung gehandelt zu haben scheint. Aufgrund der dichten späten Überbauung des Kuppenbereiches kann jedoch keine völlige Sicherheit in dieser Frage gewonnen werden. Auch die Niederlassung am Yalak Başı, für die sich ebenfalls keine antiken Befestigungen nachweisen lassen, erlebte in hellenistisch-römischer Zeit einen Aufschwung.

Der relative Wohlstand, den die Bewohner der Siedlungen während der Zeit des fortgeschrittenen Hellenismus erwerben konnten, wird durch die qualitätvollen, in Hausteintechnik errichteten Hausanlagen und teils aufwendige Sarkophage des lykischen Typs in den verschiedenen Nekropolen belegt. Nicht geklärt werden konnte allerdings die Frage nach der Gestaltung des öffentlichen Raumes in den Niederlassungen des Bonda-Gebietes in hellenistischer Zeit. Man darf jedoch davon ausgehen, daß die bedeutenderen Siedlungen über ein Amtslokal und Kultbauten verfügten. Im Siedlungsbereich dürfte die Verbauungsdichte ungleichmäßig gewesen sein; günstige Geländeterrassen waren stark verbaut, während zwischen einzelnen Baugruppen immer wieder Freiflächen bestanden.

In der in frühhellenistischer Zeit erfolgten Einrichtung eines Gürtels von Wehrdörfern im Grenzgebiet dürfte sich ein zentral gelenktes und politisch motiviertes Bemühen der Polisverwaltung von Limyra um die Organisation des westlichen Randbereiches ihres Territoriums widerspiegeln. Nebst der Grenzsicherung wurde vielleicht auch eine systematische Landnahme und eine Erweiterung der landwirtschaftlichen Nutzfläche, d.h. eine Form der Binnenkolonisation angestrebt. Hingegen darf wohl der am Ruinenbestand ablesbare Aufschwung der Dörfer des Bonda-Gebietes im fortgeschrittenen Hellenismus als Zeichen ökono- 
mischer Prosperität gewertet werden. In hellenistischem Bauzusammenhang stehende Preßanlagen, darunter auch Zwillingspressen, bieten deutliche Anzeichen für eine verkaufsorientierte Landwirtschaft.

Nebst den dörflichen Siedlungen wurden in hellenistischer Zeit auch Einzelgehöfte und Weiler errichtet: Diese lagen jedoch zumeist in einiger Entfernung von den Ortschaften und waren auf kleine, abgelegene Fruchträume ausgerichtet. Als Beispiele mögen hier etwa ein Weiler oberhalb des Gök Limanı im Küstenbereich oder das Gehöft im Trockental westlich von Finike genannt werden ${ }^{28}$. Wie ein Bau bei Yukarı Camliyurt belegt, konnten im grenznahen Bereich Gehöfte die Form von Kleinstfestungen annehmen.

In der Kaiserzeit setzten sich die im fortgeschrittenen Hellenismus angelaufenen Entwicklungstendenzen fort: Die flächenmäßige Ausdehnung der Siedlungen scheint, ebenso wie der Wohlstand ihrer Einwohnerschaft, zugenommen zu haben, Neugründungen von Niederlassungen jedoch nicht erfolgt zu sein. Da es erhebliche Schwierigkeiten bereitet, hochhellenistische und kaiserzeitliche Baubefunde zu trennen, ist es nicht möglich, genauere Angaben zur flächenmäßigen Entwicklung der Niederlassungen in diesen Perioden zu machen. Der Wohlstand der Kaiserzeit läßt sich vor allem in den Nekropolen ablesen: ein erheblicher Teil der Sarkophaggräber, zu diesen zählen auch die aufwendigsten Objekte, sind in dieser Periode entstanden. Der in den Nekropolen zur Schau gestellte Reichtum der Kaiserzeit erlaubte auch die Errichtung von Ehrenmonumenten innerhalb der Niederlassungen. Weiters wurden für die bauliche Gestaltung des öffentlichen Raumes in dieser Periode erhebliche Mittel aufgewendet: Dies wird insbesondere anhand eines an der Peripherie des Siedlungsareals gelegenen Baukomplexes am Dinek Tepesi deutlich. Zu diesem gehörten nebst einer zweiräumigen Thermenanlage auch ein Hallenbau und wahrscheinlich auch zwei direkt anschließende, großflächige Wasserspeicher.

Das Wehrdorf bei der Tespili Yayla scheint jedoch spätestens in der frühen Kaiserzeit aufgrund seiner ungünstigen, zur Zeit seiner Gründung wohl mehr aufgrund strategischer Erwägungen ausgewählten Lage weitgehend verlassen worden zu sein.

Während der antiken Siedlungsphasen bildete die Landwirtschaft die vorrangige Lebensgrundlage der dörflichen Bevölkerung. Weiters dürften - wie eine Schlackenkonzentration am Dinek Tepesi belegt - in den Dörfern auch Gewerbebetriebe angesiedelt gewesen sein. Der für die Kaiserzeit durch Grabbauten und Ehrenmonumente belegte, steigende Reichtum einiger Familien stand wohl mit einer Tendenz zur Konzentration des Grundbesitzes in Zusammenhang. Diese Entwicklung dürfte jedoch nicht zu einer Auslagerung landwirtschaftlicher Funktionen und damit zu einem Strukturwandel in der dörflichen Ökonomie geführt haben. Aus den Inschriften wird die Verschränkung der ländlichen Elite mit dem Zentralort und gleichzeitig deren Bindung an ihre Heimatgemeinden deutlich ${ }^{29}$.

${ }^{28}$ A. Konecny, Hellenistische Turmgehöfte in Zentral- und Ostlykien (1997) $15 \mathrm{ff}$.

${ }^{29}$ s. den Beitrag von $M$. Wörrle in diesem Band. 
Der Übergang von der kaiserzeitlichen zur spätantik-byzantinischen Kultur stellt sich im Denkmalbestand als fließende Entwicklung dar. Zu einem nicht genau definierbaren Zeitpunkt, wohl im späteren 4. oder dem frühen 5. Jahrhundert trat anstelle der antiken Hausteintechnik eine massive Verwendung von mörtelgebundenem Bruchstein- und Spolienmauerwerk. Die Struktur der Niederlassungen veränderte sich in dieser Periode: Im Zentrum der Siedlungen wurden große Kirchen errichtet; auch scheint die Bebauungsdichte innerhalb der Niederlassungen stark zugenommen zu haben. Zwei in mittlerer Höhe gelegene Dörfer des Bonda-Gebietes, Karakuyu und Bonda Harabesi, erreichten in dieser Periode ihre größte Flächenausdehnung. Auch in deren Umland scheint die größte Dichte an freistehenden Gehöften in spätantik-byzantinischer Zeit bestanden zu haben.

Im Gegensatz zum demographischen Aufschwung in tiefen und mittleren Siedlungslagen steht der Niedergang der Niederlassung am Dinek Tepesi: Dieses Gemeinwesen, dessen Wohlstand für die Kaiserzeit durch besonders aufwendige Grabbauten und den am Ort gepflegten Badeluxus dokumentiert ist, scheint im 4. oder im frühen 5. Jahrhundert stark an Dynamik eingebüßt zu haben. Dies wird besonders durch die Tatsache verdeutlicht, daß im Ruinenbestand kein Kirchenbau nachweisbar ist und daß die Befestigungsanlage auf der Kuppe keinerlei Hinweise auf Instandsetzungsarbeiten aufweist. Keramikfunde und vereinzelte Mörtelbruchsteinbauten belegen jedoch, daß die Niederlassung nicht völlig aufgelassen wurde. Auffälligerweise hat sich auch in Ortasarı kein Hinweis auf einen bedeutenden Kirchenbau erhalten. Dies und das Fehlen typischer Hausgrundrisse der spätantik-frühbyzantinischen Epoche können als Hinweis darauf gewertet werden, daß die Niederlassung in dieser Zeit von geringer Bedeutung gewesen sein dürfte.

Der Niedergang eines der reichsten Gemeinwesen des Bonda-Gebietes könnte mit klimatischen Veränderungen in Zusammenhang stehen, wie sie im östlichen Mittelmeer auch andernorts für diese Zeit dokumentiert sind ${ }^{30}$. Man muß wohl davon ausgehen, daß in spätantiker Zeit die wirtschaftliche Grundlage des Wohlstandes, eine Überschußproduktion im landwirtschaftlichen Bereich, wobei insbesondere Olivenöl eine tragende Rolle gespielt haben dürfte, weggebrochen ist. Eine geringfügige Zunahme von Nachtfrösten würde völlig ausgereicht haben, Ölkulturen nachhaltig zu schädigen und in der Folge einen massiven Rückgang der Produktion herbeizuführen. Die Frage, inwieweit der Niedergang des nicht erheblich höher als Karakuyu gelegenen Dorfes Ortasarı ebenfalls mit klimatischen Veränderungen in Zusammenhang gestanden hat, läßt sich nicht mit Sicherheit beantworten: Es scheint allerdings, als wäre der sicherlich nicht besonders ausgedehnte Wirtschaftsraum von Ortasar1 in Lagen zwischen 700 und $800 \mathrm{~m}$ Seehöhe befindlich gewesen. Möglich wäre es aber auch, daß die dynamische Entwicklung der nahegelegenen Niederlassung Karakuyu Auswirkungen auf die kleine Nachbargemeinde hatte.

30 s. etwa W. J. Eastwood, N. Roberts, H. F. Lamb, AnatStud 48 (1998) 77 ff.; J. Koder, Byzantina Australiensia 10 (1996) $270 \mathrm{ff}$. 
Die Instandsetzungsarbeiten an den antiken Gipfelbefestigungen von Bonda Harabesi und Ortasarı mögen im 7. oder 8. Jahrhundert oder im frühen Mittelalter ausgeführt worden sein, Sicherheit kann in dieser Frage allerdings nicht gewonnen werden. Zur selben Zeit erfolgte wohl auch die Errichtung eines Spolienmauerzuges an der Südflanke der Niederlassung am Yalak Başı.

In Lykien allenthalben feststellbare Einbauten kleiner Kirchen in bestehende basilikale Großbauten werden in mittelbyzantinische Zeit datiert. Sie gelten als Zeugnisse des wirtschaftlichen und demographischen Niedergangs im Gefolge der Araberkriege. Im Bonda-Gebiet hat sich ein derartiger Befund nur in Kirche B des Dorfes Bonda Harabesi erhalten. In Ortasarı stand ein kleinflächiger Kirchenbau im Zentrum eines an der Westseite des Siedlungshügels, am Fuß der Kuppenbefestigung gelegenen Weilers. Es scheint, als wäre die Siedlungsdichte des BondaGebietes in mittelbyzantinischer Zeit gering gewesen.

Vereinzelte Funde von Glasurkeramik belegen eine Weiternutzung der Niederlassungen des Bonda-Gebietes bis in spätbyzantinische Zeit. Dieser Periode können jedoch keine Bauaktivitäten zugeordnet werden, so daß es sich um eine Form der Subsistenzwirtschaft in den antiken Ruinen gehandelt haben mag.

In der Neuzeit fanden im Bonda-Gebiet nach Angaben aus der Bevölkerung zwei Schübe bäuerlicher Landnahme statt, im Zuge derer vier kleine Weiler und einige Einzelgehöfte errichtet wurden. Deren Bewohner scheinen ihre Existenz in erster Linie von Getreideanbau und Viehzucht gefristet zu haben. Der schlechte, steinige Boden dürfte jedoch auch diese dünne Besiedlung nicht getragen oder jedenfalls keinen ausreichenden Lebensstandard ermöglicht haben: Heute ist keiner dieser Weiler mehr permanent bewohnt und die meisten liegen wüst; anstelle der bäuerlichen Wirtschaftsform ist eine saisonale Viehwirtschaft getreten. 